\title{
Gene Expression Profiling of Broiler Liver under Cold Stress by High-Throughput Sequencing Technology
}

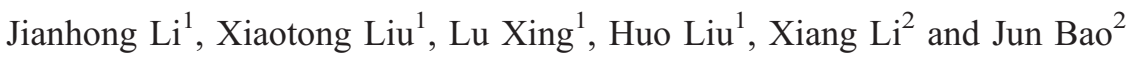 \\ ${ }^{1}$ College of Life Science and Technology, Northeast Agricultural University, Haerbin, Heilongjiang, 150030, China \\ ${ }^{2}$ College of Animal Science and Technology, Northeast Agricultural University, Harbin, Heilongjiang, 150030, China
}

\begin{abstract}
Cold stress is a major environmental factor restricting the sustainable development of animal husbandry. To gain insight into the gene-regulation processes in broilers under cold stress, gene expression profiling was conducted using high-throughput Solexa sequencing of broiler liver tissue under cold stress conditions and control conditions. According to Solexa sequencing, we identified 255 genes whose expression levels differed between the treatment and control group. Under cold stress, 135 genes were up-regulated and 120 genes were down-regulated genes compared with levels in the control group. Moreover, 469 genes were expressed only in the control group, and 172 genes were expressed only in the treatment group. These data were confirmed by real-time quantitative PCR. Gene Ontology enrichment analysis showed that the differentially expressed genes (DEGs) were mainly enriched in material metabolism and immune functions. KEGG enrichment analysis showed that DEGs were enriched in pyruvate metabolism, glycolysis/gluconeogenesis, fatty acid metabolism, insulin signaling pathway and others. In conclusion, these results may serve as an important reference for broiler breeding and provide new clues for the elucidation of molecular mechanisms of cold stress.
\end{abstract}

Key words: broiler, cold stress, gene expression, high-throughput sequencing technology, liver

\section{Introduction}

Cold stress is a major environmental factor restricting the development of animal husbandry. The chicken (Gallus gallus) can maintain its body temperature within a narrow range through physiological self-regulation. Poultry experiences cold stress when the environmental temperature suddenly drops by more than $10^{\circ} \mathrm{C}$ or when it stays at more than $4^{\circ} \mathrm{C}$ below normal temperature for an extended period (Ren and Xin, 1997). Excessive cold stress causes a series of physiological and metabolic changes in broiler chickens, such as electrolyte and acid-base imbalances, reduced endocrine function (Olanrewaju et al., 2010), and reductions in production performance including animal feed intake, body weight and feed conversion ratio (Spinua and Degena, 1993). Cold stress also disrupts the balance of the oxidant/ antioxidant system, leading to biological membrane damage and further damaging tissue integrity and negatively influencing animal health and production (Pan et al., 2005; Mujahid and Furuse, 2009). At the same time, cold stress

Received: November 17, 2016, Accepted: January 30, 2017

Released Online Advance Publication: March 25, 2017

Correspondence: Jun Bao, College of Animal Science and Technology, Northeast Agricultural University, Harbin, Heilongjiang, 150030, China.

(E-mail: Jbaoneau@sina.com) affects tissue structure lesions, inducing various types of disease (Sahin and Gumuslu, 2004). Overall, cold stress caused by low temperature has a negative influence on poultry, including slow growth, disease, and even death (Smith, 1993).

The mechanism of cold stress is highly complex. It involves a series of systems, including the nervous, endocrine and immune systems. Adaptations to mobilize various organs and tissues of the body to deal with stress and maintain relative stability primarily act through nervousendocrine pathways. However, the exact molecular mechanisms responsible for cold regulation are not well understood. Previous studies have focused on candidate genes related to cold resistance (Leandro et al., 2004; Hangalapura et al., 2006; Wang et al., 2009; Chen et al., 2012). However, many genes are key regulators of biological processes associated with cold stress, and studing the relationship between cold stress and only a few candidate genes cannot reveal the of broader picture of cold stress mechanism. Technological advances in the field of genomics, especially high-throughput next-generation sequencing, have opened new avenues of research. RNA sequencing (RNA-Seq) can reveal the presence and quantities of RNAs in a biological sample at a given moment in time. In recent years, many studies on chicken have used RNA-Seq as a powerful tool to 
compare gene expression between conditions, such as a treatment vs non-treated, and find out which genes are up- or down-regulated in each condition (Yao et al., 2011; Nie et al., 2012; Li et al., 2012; Mutryn et al., 2015; LangouetAstrie et al., 2016). In order to discover the mechanism of cold stress and identify DEGs during cold stress, gene expression at the transcriptional level was investigated using high-throughput Solexa sequencing technology. Specific genes involved in cold stress were screened and analyzed in order to reveal the mechanism of cold stress in broilers. Our results may serve as an important reference for selection and breeding of broilers and add new insight to chicken genomic studies.

\section{Materials and Methods}

\section{Chickens and Treatments}

A total of 100 1-day-old commercial AA broiler chicks were obtained from a local hatchery and housed in groups in controlled rooms with deep litter of wood shavings, construction materials, acclimatization equipment, feeders, and drinkers. The brooding temperature was maintained at $35^{\circ} \mathrm{C}$ for the first 2 days and then reduced gradually to $29^{\circ} \mathrm{C}$ until 14day of age.

At 15 days of age, 24 broiler chicks were chosen randomly from 100 broiler chicks and divided into two groups, the control group and treatment group, each with 3 replicates (4 chicks per replicate). The control group was kept in the normal thermal environment, in which from 15 days of age to 28 days of age, the temperature was gradually reduced from $29^{\circ} \mathrm{C}$ to $23^{\circ} \mathrm{C}$. The temperature of the treatment group was $5^{\circ} \mathrm{C}$ lower than the normal temperature for 6 hours. Treatment chickens were exposed to cold stress from 15 days of age to 28 days of age. Birds were reared on battery cages (4 birds per cage) until slaughter. The density of birds was $10 / \mathrm{m}^{2}$.

Broilers received a commercial starter diet $(12.10 \mathrm{MJ} / \mathrm{kg}$ metabolizable energy and $22 \%$ crude protein) until 7 days of age, after which a commercial grower diet $(12.68 \mathrm{MJ} / \mathrm{kg}$ metabolizable energy and $20 \%$ crude protein) was provided until the end of the experiment. The light regime was 23L: 1D for the first 3days and then 16L:8D light program was applied. Birds had free access to feed and water during the rearing period.

All procedures performed in the present study were approved by the Institutional Animal Care and Use Committee of Northeast Agriculture University.

\section{Sample Preparation and Total RNA Extraction}

At 28 days of age, the liver tissues of the treatment chickens and control birds were obtained for gene expression analysis. To obtain complete gene expression profiles, each sample was assayed with three biological replicates (1 chick per replicate). The collected tissue samples were immediately frozen in liquid nitrogen and stored at $-80^{\circ} \mathrm{C}$ until further use.

Total RNA was isolated from the liver tissue samples of broilers separately using RNAiso Plus kit (TaKaRa, Dalian, China) according to the manufacturer's protocol and RNase- free DNaseI was used to remove DNA contamination. Dried RNA samples were dissolved in diethylpyrocarbonatetreated $\mathrm{H}_{2} \mathrm{O}$. RNA integrity was verified by agarose gel electrophoresis and visualisation of the $28 \mathrm{~S}$ and $18 \mathrm{~S}$ ribosomal RNA.

The quality of the RNA was checked by Agilent 2100 biological analyzer (Agilent, Santa Clara, CA, USA). The concentration of the RNA samples was determined using Nano Drop-2000 (Thermo, USA).

\section{Library Construction and Deep Sequencing}

The main reagents and supplies are Illumina Gene Expression Sample Prep Kit and Illumina Sequencing Chip, and the main instruments are Illumina Cluster Station and Illumina HiSeqTM 2000 System. At least $6 \mu \mathrm{g}$ of total RNA $(\geq 300 \mathrm{ng} / \mu l)$ was prepared and sent to Beijing Genomics Institute for Solexa sequencing. The mRNA was purified from total RNA using a Oligo (dT) magnetic bead, and then the first and second-strand complementary DNA were synthesized. The bead-bound complementary DNA was subsequently digested with the restriction enzyme NlaIII, which recognizes and removes the CATG sites. The fragments without the $3^{\prime}$ complementary DNA fragments connected to Oligo (dT) beads were washed away and the Illumina adaptor 1 (sense: 5' ACACTCTTTCCCTACACGACGCTCT TCCGATC $3^{\prime}$ ) was ligated to the cohesive $5^{\prime}$ end of the digested bead-bound complementary DNA fragments. The junction of Illumina adaptor 1 and CATG site was the recognition site for Mme I, which is a type of endonuclease that has separate sites for recognition and digestion. It cut at a site $17 \mathrm{bp}$ downstream from the CATG site. After removing the 3' fragments with magnetic bead precipitation, the Illumina adaptor 2 (sense: 5' GATCGGAAGAGCGGTTCAGCAGG AATGCCGAG3' ) was ligated to the $3^{\prime}$ ends of tags, producing tags with different adaptors at both ends to form a tag library. The library was amplified by PCR for 15 cycles, and $105 \mathrm{bp}$ fragments were purified in $6 \%$ TBE PAGE gel electrophoresis. After denaturation, the single-chain molecules were fixed onto the Illumina Sequencing chip. Each molecule grew into a single-molecule cluster sequencing template through in situ amplification. Four different nucleotides were labeled with four different fluorophores, and then the samples were sequenced by synthesis. During this process, adaptor 1 was used as sequencing primer.

\section{Analysis and Mapping of DGE Tags}

To map the Digital Gene Expression Profiling (DGE) tags, the sequenced raw data were filtered to remove low quality tags (tags with unknown nucleotide "N"), empty tags (no tag sequence between the adaptors) and tags with only one copy number (which might result from sequencing errors). For tags annotation, the clean tags containing CATG and $21 \mathrm{bp}$ tag sequences were mapped to database of chicken (Gallus gallus) genome sequence, allowing no more than one nucleotide mismatch. The clean tags mapped to reference sequences from multiple genes were filtered. The remaining clean tags were designed to be unambiguous. For gene expression analysis, the number of unambiguous clean tags for each gene was calculated and normalized to TPM (num- 
Table 1. Specific amplification of the gene and internal reference primer

\begin{tabular}{lll}
\hline \hline Gene & \multicolumn{1}{c}{ Forward primer $\left(5^{\prime}-3^{\prime}\right)$} & \multicolumn{1}{c}{ Reverse primer $\left(5^{\prime}-3^{\prime}\right)$} \\
\hline$H S P 70$ & CACCTGCAGAAGCAGCCATAA & TTATGAACACTGCTATGCCACACAA \\
$P P A R-\alpha$ & TGCACTGGAACTGGATGATAGTGA & TCCTACATTTACAAGACCAGGACGA \\
CRHR2 & GGACATGGGCCTCCAAGATAAAC & GATGAGCCCAATCTTGTAATGGTG \\
$M X$ & GAGTACCTTCAGCCTGTTT & GCTGGTCAGTAACTTCTGCT \\
$L P L$ & AGTCAGAGTGAAGTCAGGCGAAAC & CTGCTCCAGGCACTTCACAAATA \\
$F A S$ & ATGTCGTTCATGTGACAAAGCACTC & GTACATGACTCGCAATGTTCACACC \\
GAL2 & GCGAATTCCATGAGGATTCTTACC & GCTCTAGATAATGCATTCCAAGGC \\
$R P L 15$ & TGCTGGCAGTAGCGTTCAGG & ACGCATTTCCCTGTGCTT \\
$\beta$-actin & ATTGTCCACCGCAAATGCTTC & AAATAAAGCCATGCCAATCTCGTC \\
\hline
\end{tabular}

ber of transcripts per million clean tags) (Morrissy et al., 2009).

To compare the differences in gene expression, the tag frequency in each DGE library was statistically analyzed according to the method described by Audic and Claverie (Audic and Claverie, 1997). For gene expression variance, T-testing was performed to determine significant differences. The $\mathrm{P}$ values corresponding to differential gene expression were assessed. The false discovery rate (FDR) method was used to determine the threshold of $\mathrm{P}$ value through a multiple test and analysis as per Benjamini (Benjamini and Hochberg, 1995). We used FDR $\leq 0.001$ and the absolute value of $\log _{2}$ ratio $>1$ as the threshold to judge the significant differences in gene expression.

To gain insight into the biological roles of these DEGs, we performed Gene Ontology (GO) annotation and functional classification analysis. To examine the biological significance of DEGs, GO enrichment analysis was used to investigate functional distributions by mapping each DEG to terms in the GO database (http://www.geneontology.org). In addition, we also performed pathway enrichment analysis based on the Kyoto Encyclopedia of Genes and Genomes (KEGG) database (http://www.genome.jp/KEGG). For enrichment analysis, all $\mathrm{P}$-values were subjected to Bonferroni correction. We selected a corrected P-value $<0.05$ as a threshold to determine significant enrichment of the gene sets.

\section{Real-Time Quantitative RT-PCR (qRT-PCR) Analysis}

To test the reliability of Solexa sequencing, the expression of the eight candidate genes was determined using quantitative reverse transcription-PCR (qRT-PCR). The reaction was performed using SYBR ${ }^{\circledR}$ Premix Ex Taq $^{\text {TM }}$ II Perfect Real-Time kit (TaKaRa). Primers were designed based on the target gene sequences using the Primer Premier 5.0 software (Premier Biosoft International, PaloAlto, CA) and then were synthesized commercially (TaKaRa Biological Engineering Co., Ltd., Dalian, China). The primers are listed in Table 1. cDNA was synthesized from total RNA using PrimeScript ${ }^{\circledR}$ RT reagent Kit (TaKaRa). For each sample 2 $\mu \mathrm{L}$ of cDNA was used as a template in a $20 \mu \mathrm{L}$ reaction. The thermal cycling conditions had an initial denaturation step at $95^{\circ} \mathrm{C}$ for $1 \mathrm{~min}$, followed by 40 cycles including denaturation step at $95^{\circ} \mathrm{C}$ for $5 \mathrm{~s}$, and annealing and extension step at 60

\section{${ }^{\circ} \mathrm{C}$ for $34 \mathrm{~s}$.}

The $\beta$-actin gene was used as an internal control. Each experiment was performed in triplicate and repeated three times independently. The cycle threshold $(\mathrm{Ct})$ values of the triplicate PCRs were averaged and relative quantification of the transcript levels was performed using the comparative $2^{-\Delta \Delta \mathrm{CT}}$ method (Livak and Schmittgen, 2001). The fold change in the target gene relative to $\beta$-actin was determined with the following formula: fold change $=\Delta \Delta \mathrm{CT}$, where $\Delta \Delta \mathrm{CT}=(\mathrm{Ct}$ target gene $-\mathrm{Ct} \beta$-actin $)$. Each sample was further amplified without reverse transcription to confirm that no DNA contamination was in the sample.

\section{Statistical Analysis}

Data of qRT-PCR were analysed using SAS statistical software, version 9.1. The results were expressed as mean \pm SEM. The process of Univariate was used for testing the normality of data. T-test was used for comparing the means of two groups (control and treatment group).

\section{Results}

\section{Solexa Sequencing}

After filtering the dirty tags (Tags containing $\mathrm{N}$, only adaptors and copy number $<2$ ), we generated 3646672 reads for the control group and 3774114 reads for the treatment group using Solexa sequencing. After filtering out low-complexity, low-quality and repetitive tags, 355031 clean tags for the control group and 361904 clean tags for the treatment group were obtained. Mapping to the database of chicken genome sequence, 10233 genes for the control group and 9936 genes for the treatment group were annotated. Moreover, 469 genes were found to be expressed only in the control group, and 172 genes were expressed only in the cold stress group with 9764 genes expressed in both groups. We used FDR $\leq 0.001$ and an absolute value of $\log _{2}$ ratio $>1$ as the threshold to determine the significant differences in gene expression. We identified 255 genes whose expression levels differed between the treatment and control group. Under cold stress, 135 genes were identified as up-regulated genes and 120 genes were down-regulated compared with levels in control animals. The results of Solexa sequencing are presented in Table 2 and Fig. 1. 
Table 2. The total number of sequencing tags obtained from each sample

\begin{tabular}{cc}
\hline \hline Gene & Number \\
\hline Total reads & $\begin{array}{l}\text { control group: } 3646672 \\
\text { cold stress group: } 3774114\end{array}$ \\
\hline Clean Tags & $\begin{array}{c}\text { control group: } 355031 \\
\text { cold stress group: } 361904\end{array}$ \\
\hline Annotated genes & control group: 10223 \\
& cold stress group:9936 \\
\hline differential expression genes & 255 \\
up-regulated genes & 135 \\
down-regulated genes & 420 \\
only in the control group & 172 \\
only in the cold stress group & 9764 \\
in both group &
\end{tabular}

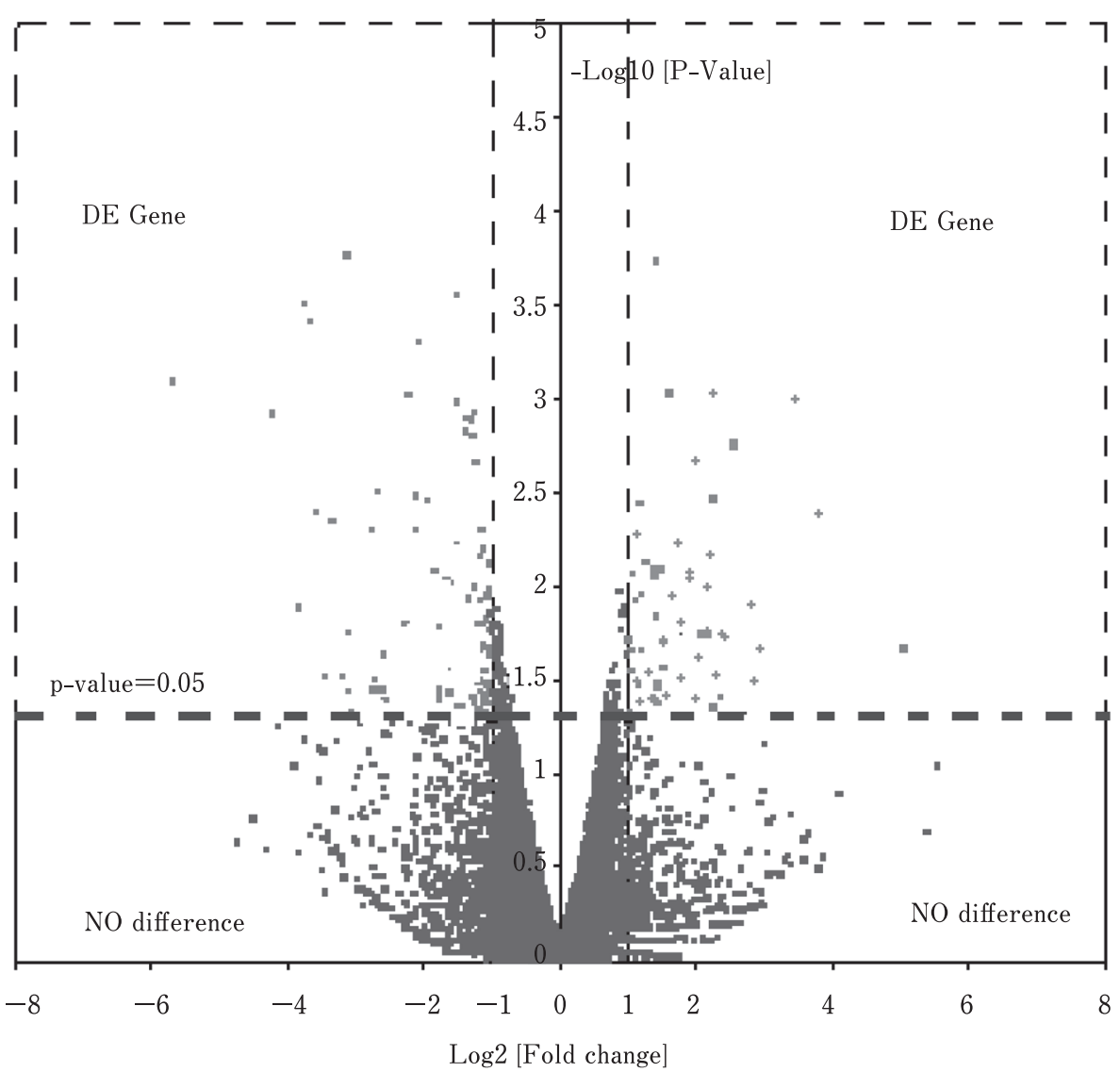

Fig. 1. The volcano plot about DEGs. A volcano plot is constructed by plotting the negative log of the $\mathrm{p}$ value on the $\mathrm{y}$ axis (base 10). The $\mathrm{x}$ axis is the log of the fold change between treatment group and control group. The horizontal dotted line shows where $p$ value $=0.05$ with points above the line having $\mathrm{p}$ value $<0.05$ and points below the line having $\mathrm{p}$ value $>0.05$. The two vertical dotted lines represent 2 fold changes. The differentially expressed genes are shown in the dotted box of Fig. 1. Up-regulated genes are shown in the right side and downregulated genes are shown in the left side. Genes without differential expressions are shown outside of the dotted box in Fig. 1. 


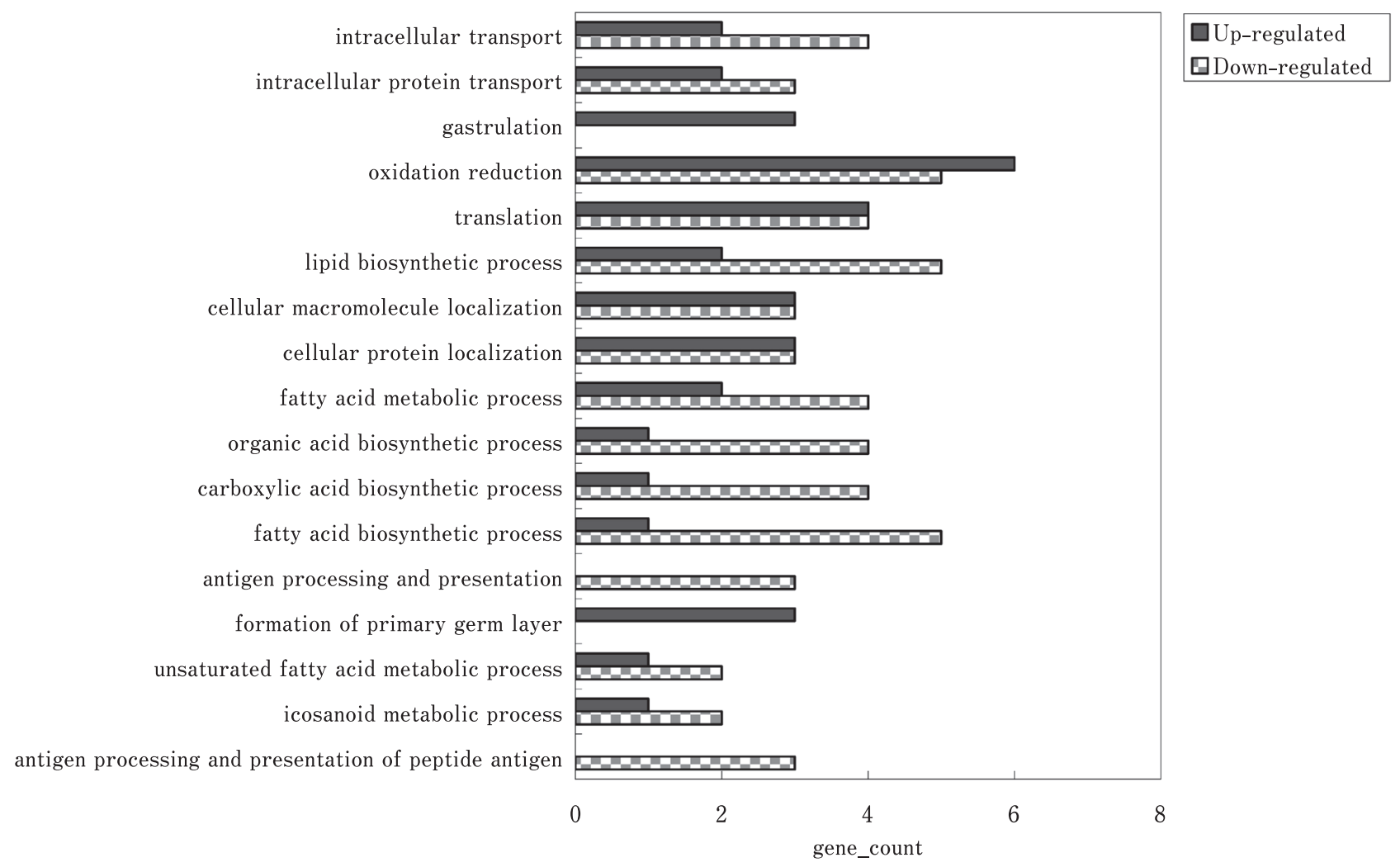

Fig 2-a. Biological processes.

\section{Functional Classification of the Differential Expression Genes}

Gene ontology (GO) is an internationally standardized gene function classification system for comprehensively describing the properties of genes and their products in any organism. GO has three ontologies: molecular functions, cellular components, and biological processes. DEGs were classified into $99 \mathrm{GO}$ categories. Among these categories, $24 \mathrm{GO}$ categories belonged to the biological process ontology (Fig 2-a), 33 GO categories belonged to the cellular component ontology (Fig 2-b), and 42 GO categories belonged to the molecular function ontology and (Fig 2-c).

$\mathrm{GO}$ enrichment analysis was performed, and the results are listed in Table 3. All processes listed had enrichment Pvalues $<0.05$. The significantly enriched GO terms of DEGs include fatty acid metabolic process, fatty acid biosynthetic process, lipid biosynthetic process, antigen processing and presentation of peptide antigen, carboxylic acid biosynthetic process, organic acid biosynthetic process, translation, cellular protein localization, icosanoid metabolic process, cellular macromolecule localization and so on.

KEGG analysis identified the potential involvement of transcripts in biological pathways. The most enriched pathways included pyruvate metabolism, fatty acid metabolism, insulin signaling pathway and so on. Enriched KEGG terms of DEGs are presented in Table 4.

\section{Confirmation of Solexa Expression Patterns by $q R T-P C R$ Analyses}

qRT-PCR was applied to validate the expression patterns of differentially expressed genes. Eight genes were selected including 3 up-regulated expression genes ( $L P L, G A L 2$, $R P L 15)$ and 5 down -regulated expression genes (HSP 70, PPAR- $\alpha, C R H R 2, M X, F A S)$. The results by qRT-PCR show that the 8 selected genes had the same expression patterns as Solexa sequencing (Fig. 3 and Table 5). We analyzed the correlation between the FPKM values obtained by RNA-seq with their corresponding qRT-PCR Ct values; the two values represent the quantitative levels of expression of a specific transcript in the RNA sample. The $\mathrm{Ct}$ values from three biologic replicates were compared to the corresponding $\log _{2}$ FPKM values. The results showed that $\log _{2}$ FPKM values from RNA-seq analysis have a strong linear correlation with $\mathrm{Ct}$ values from qRT-PCR, with a relative coefficient of $\mathrm{R}^{2}=$ 0.9631 (Fig. 4). This data indicate that original Solexa analysis is validated in the differentially expressed genes.

\section{Discussion}

Temperature plays an important role in the growth and development of animals. Exposure to a cold environment causes a series of physiological reactions to produce more heat to maintain body temperature, including the use of adipose tissue in most animals. In poultry, however, the liver rather than adipose tissue, plays a key role in lipid metabo- 


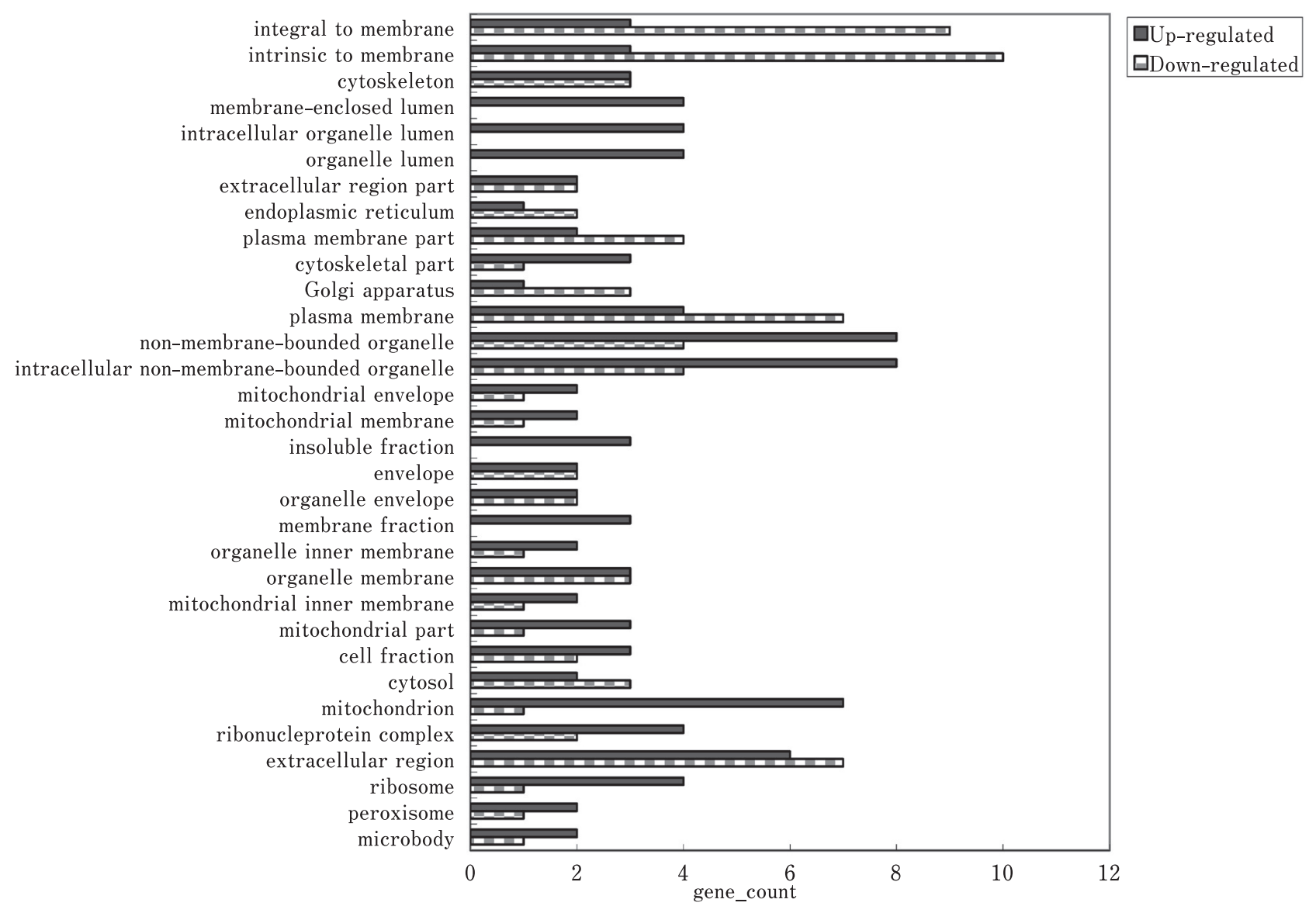

Fig 2-b. Cellular Components.

lism. Fatty acid synthesis in poultry also mainly occurs in the liver rather than in fat tissue (Nguyen et al., 2008). Therefore, the liver is a vital organ for studying the effect of cold stress on the body in poultry. In this study, gene expression profiling in the liver of broiler chicken under cold stress was conducted through the use of high throughput sequencing technology in order to reveal the molecular mechanisms involved in the response to cold stress.

The experimental results showed that 255 DEGs were identified, including 135 up-regulated genes and 120 downregulated genes in chickens exposed to cold stress. Chen et al. (2014) used high-throughput Solexa sequencing technology to identify DEGs in the chicken hypothalamus during cold stress and found 334 down-regulated genes and 543 upregulated genes. Although the design of the two experiments differed and the tissues obtained for gene expression analysis are different, both experiments revealed that the expression levels of many genes are altered by cold stress. These results indicate that cold stress has an effect on the expression of many specific genes. These genes function is different in response to cold stress. Thus, it appears that the molecular mechanisms of responses to cold stress in broilers are highly complex, involving a series of physiological and biochemical changes and the involvement of many genes. The reasons behind DEG expression level changes will require further research. The findings of this study will be useful in the further investigation of the cold-specific signaling networks in broilers.

In addition to the identified DEGs, 172 genes were expressed only in the cold stress group. These results indicate that the expression of some genes was activated by cold stress. It seems that many genes, including several cold resistance genes, are not expressed under normal conditions. Genes induced by cold stress are likely to be involved in responses to cold. Animals adapt to adverse conditions by promoting the expression of these genes. These genes may be specific to defense against cold stress and are attractive targets for further functional characterization. Similarly, 469 genes were expressed only in the control group. The results showed that the expression of some gene was repressed under the cold stress conditions in order to better enable the synthesis of proteins associated with cold resistance.

GO enrichment analysis of DEGs revealed genes related to biological functions. For further discussion, we will focus on GO terms that were highly significant $(P<0.01)$. The results of GO analysis showed that DEGs mainly enriched in two systems: material metabolism and immune function. 


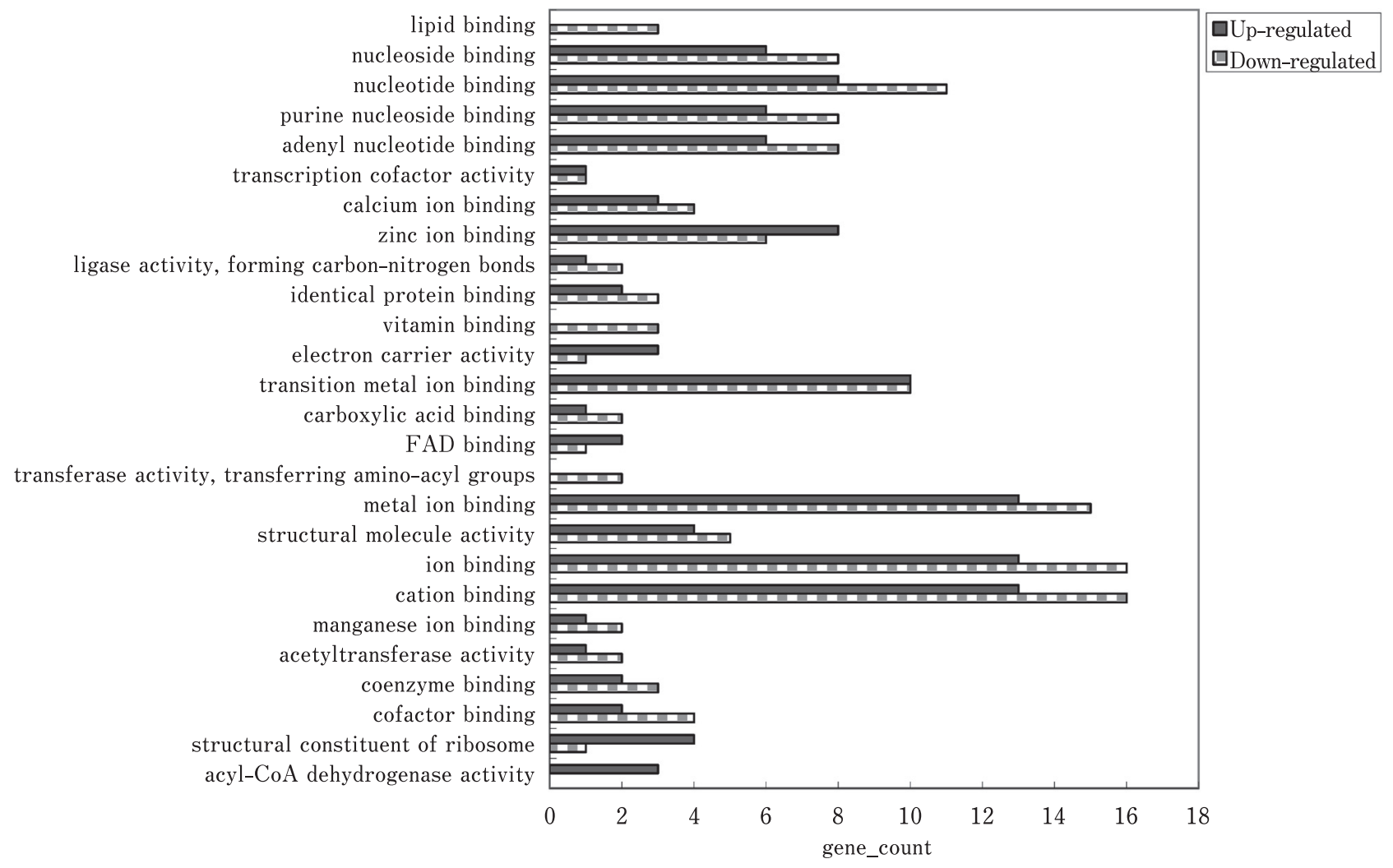

Fig 2-c. Molecular Function.

Fig. 2. GO analysis of differentially expressed genes.

(Note: GO categories which contain more than 3 genes was displayed in figure Fig. 2)

Two genes that were up-regulated under cold stress are $A C O X 1$ and $L T A 4 H$, which are involved in material metabolism. The first rate-limiting oxidation step in the peroxisomal pathway is catalyzed by acyl-CoA oxidase 1 . The upregulation of $A C O X 1$ gene reflects the fact that the $\beta$ oxidation of fatty acids in peroxisomes was strengthened by cold stress. $L T A 4 H$ gene encodes leukotriene A4 hydrolase which catalyzes the final step in the synthesis of leukotriene B4 (LTB4). The leukotrienes B4 are important lipid mediators with immune modulatory and pro-inflammatory properties. Studies have shown that $L T A 4 H$ mutations result in hypersusceptibility to mycobacterial infection (Tobin et al., 2010). The up-regulation of $L T A 4 H$ indicates that it may be involved in inflammation and the immune response in chickens under cold stress.

Five genes that were down-regulated under cold stress are $A C A C A, S C D, F A S N, I D I 1$, and $C D 74$, which are involved in material metabolism. $A C A C A$ encodes acetyl CoA carboxylase which catalyzes the rate-limiting carboxylation of acetyl CoA to malonyl CoA during long-chain fatty acid synthesis. FASN encodes fatty acid synthase which catalyzes the synthesis of palmitate from acetyl-CoA and malonyl-CoA in the presence of NADPH into long-chain saturated fatty acids. $S C D$ encodes stearoyl-CoA desaturase, which is a key enzyme that catalyzes a rate-limiting step in the synthesis of unsaturated fatty acids. SCD-deficient mice show reduced TAG accumulation in white adipose tissue (Miyazaki et al., 2009). These results suggest that $S C D$ might have an important role in providing substrate for triacylglycerols (TAGs) synthesis. IDI1 encodes a peroxisomally localized enzyme that catalyzes the interconversion of isopentenyl diphosphate (IPP) to its highly electrophilic isomer, dimethylallyl diphosphate (DMAPP), which is the substrate for the subsequent reaction that results in the synthesis of cholesterol. Adipose tissue is a key organ in the regulation of body energy homeostasis, displaying a great plasticity (Rosen and Spiegelman, 2006). Fatty acids are either esterified and stored as TAGs or $\beta$-oxidized and used as energy, according to the energy demands of the organism. Several studies have shown that fatty acids can regulate their own metabolism, acting at the gene transcription level (Sampath and Ntambi, 2005). Some transcription factors are prospective fatty acid targets, regulating the expression of enzymes involved in lipid metabolism (Jump et al., 1994, 2005). CD74 is also known as a major histocompatibility complex class II molecule (MHC II). MHC II plays an essential role in glucose and lipid metabolism. Research has shown that compared with wild-type mice, $M H C I I$ knockout mice fed a high-fat diet 
Table 3. Enriched GO terms in differentially expressed genes

\begin{tabular}{|c|c|c|c|c|c|}
\hline \multirow{2}{*}{ Term } & \multirow{2}{*}{ Description } & \multirow{2}{*}{ Count } & \multicolumn{2}{|c|}{ Genes } & \multirow{2}{*}{ PValue } \\
\hline & & & Up & Down & \\
\hline GO:0006631 & $\begin{array}{c}\text { fatty acid metabolic } \\
\text { process }\end{array}$ & 6 & ACOX1, LTA4H & $\begin{array}{l}S C D, A C A C A \\
F A S N, C D 74\end{array}$ & $3.41 \mathrm{E}-04$ \\
\hline GO:0006633 & $\begin{array}{c}\text { fatty acid biosynthetic } \\
\text { process }\end{array}$ & 5 & $L T A 4 H$ & $\begin{array}{l}S C D, A C A C A \\
F A S N, C D 74\end{array}$ & $3.77 \mathrm{E}-04$ \\
\hline GO:0008610 & $\begin{array}{l}\text { lipid biosynthetic } \\
\text { process }\end{array}$ & 7 & ACOX1, LTA4H & $\begin{array}{c}S C D, A C A C A, \\
F A S N, I D I 1, C D 74\end{array}$ & $6.87 \mathrm{E}-04$ \\
\hline GO:0048002 & $\begin{array}{l}\text { antigen processing } \\
\text { and presentation of } \\
\text { peptide antigen }\end{array}$ & 3 & & $T A P 2, B F 2, C D 74$ & 0.004169 \\
\hline GO:0046394 & $\begin{array}{c}\text { carboxylic acid } \\
\text { biosynthetic process }\end{array}$ & 5 & $L T A 4 H$ & $\begin{array}{l}S C D, A C A C A \\
F A S N, C D 74\end{array}$ & 0.005184 \\
\hline GO:0016053 & $\begin{array}{c}\text { organic acid } \\
\text { biosynthetic process }\end{array}$ & 5 & $L T A 4 H$ & $\begin{array}{l}S C D, A C A C A \\
F A S N, C D 74\end{array}$ & 0.005184 \\
\hline GO:0006690 & $\begin{array}{l}\text { icosanoid metabolic } \\
\text { process }\end{array}$ & 3 & $A C O X 1, L T A 4 H$ & $C D 74$ & 0.007991 \\
\hline GO:0033559 & $\begin{array}{l}\text { unsaturated fatty acid } \\
\text { metabolic process }\end{array}$ & 3 & $A C O X 1, L T A 4 H$ & $C D 74$ & 0.007991 \\
\hline GO:0006412 & translation & 8 & $\begin{array}{l}\text { RPL15, RPLP1, } \\
\text { MRPL51, RPS8 }\end{array}$ & $\begin{array}{l}\text { TARS, EIF4A2, } \\
\text { EPRS, RPL17 }\end{array}$ & 0.01675 \\
\hline GO:0055114 & oxidation reduction & 11 & $\begin{array}{c}\text { LDHB, ACOX1, } \\
\text { CYP } 2 C 18, \\
\text { LOC417013, } \\
\text { ACAD11 }\end{array}$ & $\begin{array}{l}A L D H 2, F A S N, M E 1, \\
X D H, N D U F V 2, S C D\end{array}$ & 0.011213 \\
\hline GO:0001704 & $\begin{array}{c}\text { formation of primary } \\
\text { germ layer }\end{array}$ & 3 & $\begin{array}{c}\text { PRKARIA, SMAD1, } \\
\text { CTNNB1 }\end{array}$ & & 0.028138 \\
\hline GO:0034613 & $\begin{array}{l}\text { cellular protein } \\
\text { localization }\end{array}$ & 6 & $\begin{array}{c}\text { LOC769383, } \\
\text { TIMM17A, CTNNB1 }\end{array}$ & CD74, COPA, SRP14 & 0.029322 \\
\hline GO:0070727 & $\begin{array}{c}\text { cellular macromolecule } \\
\text { localization }\end{array}$ & 6 & $\begin{array}{c}\text { LOC769383, } \\
\text { TIMM17A, CTNNB1 }\end{array}$ & COPA, CD74, SRP14 & 0.030944 \\
\hline GO:0019882 & $\begin{array}{l}\text { antigen processing } \\
\text { and presentation }\end{array}$ & 3 & & $B F 2, C D 74, T A P 2$, & 0.047878 \\
\hline GO:0042579 & microbody & 3 & $A C O X 1, A C A D 11$ & $\mathrm{XDH}$ & 0.015945 \\
\hline GO:0005777 & peroxisome & 3 & $A C O X 1, A C A D 11$ & $\mathrm{XDH}$ & 0.015945 \\
\hline GO:0003995 & $\begin{array}{c}\text { acyl-CoA } \\
\text { dehydrogenase activity }\end{array}$ & 3 & $\begin{array}{c}A C O X 1, A C A D 11 \\
L O C 417013\end{array}$ & & 0.010476 \\
\hline GO:0003735 & $\begin{array}{l}\text { structural constituent } \\
\text { of ribosome }\end{array}$ & 5 & $\begin{array}{c}M R P L 51, R P L 15, \\
\text { RPLP1, RPS }\end{array}$ & RPL17 & 0.032122 \\
\hline GO:0048037 & cofactor binding & 6 & $A C O X 1, A C A D 11$ & $\begin{array}{c}X D H, M E 1 \\
F A S N, N D U F V 2\end{array}$ & 0.038821 \\
\hline GO:0050662 & coenzyme binding & 5 & $A C O X 1, A C A D 11$ & $\begin{array}{l}M E 1, X D H \\
\text { NDUFV2 }\end{array}$ & 0.043767 \\
\hline GO:0016407 & $\begin{array}{l}\text { acetyltransferase } \\
\text { activity }\end{array}$ & 3 & SAT1 & $F A S N, P C A F$ & 0.047696 \\
\hline
\end{tabular}


Table 4. Enriched KEGG terms in differentially expressed genes

\begin{tabular}{|c|c|c|c|c|c|}
\hline \multirow{2}{*}{ Term } & \multirow{2}{*}{ Description } & \multirow{2}{*}{ Count } & \multicolumn{2}{|c|}{ Genes } & \multirow{2}{*}{ PValue } \\
\hline & & & Up & Down & \\
\hline gga00620 & Pyruvate metabolism & 6 & $\begin{array}{c}A C O X 1, A L D H 2 \\
H A D H B\end{array}$ & $M E 1, A C A C A, F A S N$ & $7.84 \mathrm{E}-05$ \\
\hline gga00071 & Fatty acid metabolism & 5 & $\begin{array}{c}A C O X 1, A C A A 2 \\
A L D H 2, A C A T 2, \\
H A D H B\end{array}$ & & $6.11 \mathrm{E}-04$ \\
\hline gga00010 & $\begin{array}{c}\text { Glycolysis / } \\
\text { Gluconeogenesis }\end{array}$ & 4 & $L D H B, F B P 1, P C K 1$ & $P G M 1$ & 0.003024 \\
\hline gga00640 & Propanoate metabolism & 4 & $\begin{array}{c}L D H B, A L D H 2, \\
\quad A C A T 2\end{array}$ & $A C A C A$, & 0.005168 \\
\hline gga00280 & $\begin{array}{c}\text { Valine, leucine and } \\
\text { isoleucine degradation }\end{array}$ & 3 & $\begin{array}{c}A C A T 2, H A D H B \\
\text { and } A C A A 2\end{array}$ & & 0.016075 \\
\hline gga03010 & Ribosome & 4 & $\begin{array}{l}M R P L 51, R P L 15, \\
R P L P 1, R P L P 2,\end{array}$ & & 0.018094 \\
\hline gga04910 & $\begin{array}{c}\text { Insulin signaling } \\
\text { pathway }\end{array}$ & 6 & $\begin{array}{c}F B P 1, P C K 1 \\
\text { PRKAR1A }\end{array}$ & $\begin{array}{c}A C A C A, F A S N, \\
\text { SOCS1 }\end{array}$ & 0.019435 \\
\hline gga00061 & Fatty acid biosynthesis & 4 & & $\begin{array}{c}A C A C A, F A S N \\
S C D, I D I 1\end{array}$ & 0.072994 \\
\hline gga00062 & $\begin{array}{l}\text { Fatty acid elongation } \\
\text { in mitochondria }\end{array}$ & 4 & $\begin{array}{c}A C O X 1, \\
A C A A 2, H A D H B\end{array}$ & $A C A C A$ & 0.086954 \\
\hline
\end{tabular}

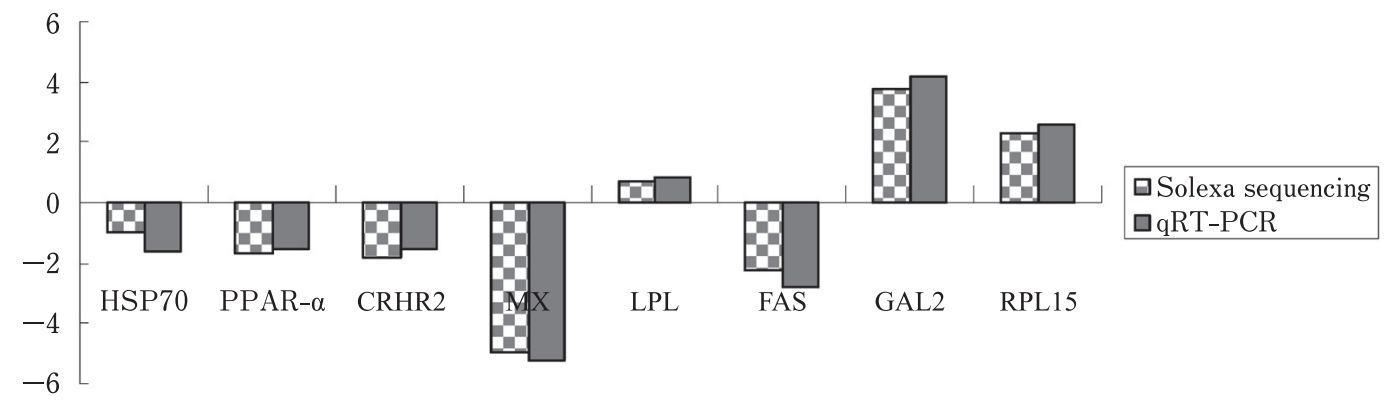

Fig. 3. Comparison of the expression of eight selected genes using Solexa sequencing and qRT-PCR.

have low blood glucose, insulin and free fatty acid levels (Cho et al., 2014).

Based on our results, we speculate that the downregulation of $A C A, F A S N$, and $S C D$ genes causes a decrease in the activity of these enzymes, indicating that fatty acid synthesis was repressed and fatty acid $\beta$-oxidation was enhanced under cold stress. The down-regulated expression of $C D 74$ reflects that the oxidation reactions of fatty acid $\beta$-oxidation and glucose were strengthened to provide more energy under cold stress. Moreover, the down-regulation of IDI1 acts to decrease cholesterol biosynthesis. These results show that chickens under cold stress increase metabolic heat production to maintain body temperature by promoting fatty acid oxidation and, at the same time, inhibiting fatty acid and cholesterol synthesis to reduce the use of acetyl coenzyme A. This allows more acetyl coenzyme A to enter the Krebs cycle, releasing more energy.

Immune functions include antigen processing and presentation and antigen processing and presentation of peptide antigen. Three genes involved in immune function were down-regulated in chickens under cold stress: TAP2, BF2 and CD74. TAP2 and BF2 (also known as an MHC class I molecule) are involved in antigen presentation by $\mathrm{MHC}$ class I molecules, especially in the transport of endogenous peptides. These transport proteins play important roles in antigen processing and presentation (Jensen, 2007). The $B F 2$ gene is dominantly expressed and confers resistance to certain poultry diseases. In chickens, the dominantly expres- 
Table 5. Comparison of mRNA expression level of DGEs in the treatment group and control group

\begin{tabular}{cccc}
\hline \hline GENE & Treatment group & Control group & P Value \\
\hline HSP70 & $1.86 \pm 0.94$ & $14.77 \pm 2.83$ & $<0.01$ \\
PPAR- $\alpha$ & $1.52 \pm 0.32$ & $10.16 \pm 1.67$ & $<0.01$ \\
CRHR2 & $1.50 \pm 0.35$ & $8.46 \pm 1.01$ & 0.0263 \\
MX & $5.21 \pm 1.24$ & $13.47 \pm 2.32$ & 0.014 \\
LPL & $0.85 \pm 0.31$ & $0.42 \pm 0.18$ & 0.0218 \\
FAS & $2.81 \pm 0.53$ & $31.43 \pm 1.98$ & $<0.01$ \\
GAL2 & $4.20 \pm 1.12$ & $1.64 \pm 0.52$ & 0.016 \\
RPL15 & $2.56 \pm 0.96$ & $0.97 \pm 0.22$ & 0.0206 \\
\hline
\end{tabular}

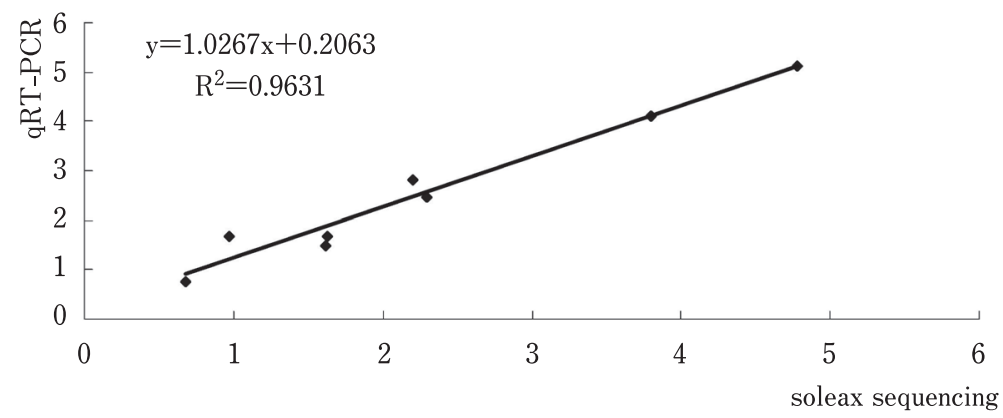

Fig. 4. Pearson correlation scatter plot between Solexa sequencing and qRT-PCR.

sed $B F 2$ locus determines the immune response to certain infectious pathogens and even vaccine efficacy (Kaufman, 2000). CD74 plays a major role in the processing of $\mathrm{MHC}$ class II molecules in antigen-presenting immune cells (Stumptner-Cuvelette and Benaroch, 2002). Recently, a novel role for the cell surface molecule $C D 74$ has been identified as part of a receptor complex with CD44, which interacts with the cytokine macrophage migration inhibitory factors MIF3 and MIF4. MIF has important roles in the innate and acquired immune responses (Leng et al., 2003). Levels of antigen presentation to the surface of cells would be reduced when gene expression levels of TAP2, BF2 and CD74 decreased under cold stress, weakening the body's immunity to diseases. These results are consistent with the common phenomenon that the body is most likely to get sick under cold stress.

In order to further understand the gene regulatory mechanisms of cold stress, KEGG pathway analysis of DEGs was performed. For further discussion, we choose pathway which reached very significant level to discussion $(p<0.01)$. The results show that DEGs were enriched in pathways associated with metabolism.

Four genes included $L D H B, F B P 1, P C K 1$, and $P G M 1$ are involved in the pathway of glycolysis/gluconeogenesis pathway. Of these, $L D H B, F B P 1$, and $P C K 1$ were up-regulated and $P G M 1$ was down-regulated under cold stress. FBP1 gene encodes a gluconeogenesis regulatory enzyme that catalyzes the hydrolysis of fructose 1,6-bisphosphate to fructose 6-phosphate and inorganic phosphate. Fructose-1,6diphosphatase deficiency is associated with hypoglycemia and metabolic acidosis (Baker and Winegrad,1970). PCK1 encodes phosphoenolpyruvate carboxykinase 1 , which catalyzes the formation of phosphoenolpyruvate from oxaloacetate, releasing carbon dioxide and GDP. Defect in this gene cause cytosolic phosphoenolpyruvate carboxykinase deficiency (Hommes et al., 1976). LDHB encodes lactate dehydrogenase $\mathrm{B}$, which catalyzes the reversible conversion of lactate and pyruvate, as well as NAD and NADH, in the glycolytic pathway. Mutations in this gene are associated with lactate dehydrogenase B deficiency. PGMI encodes phosphoglucomutase 1 , which catalyzes the transfer of phosphate between the 1 and 6 positions of glucose. FBPase and phosphoenolpyruvate carboxykinase 1 are the main control points for the regulation of gluconeogenesis. Cold stressinduced transcription of PCK1 and FBP1 helps that maintain blood sugar levels by promoting gluconeogenesis, resulting in the inhibition of glycolysis and stimulation of gluconeogenesis.

Six genes were found to be involved in pyruvate metabolism, propanoate metabolism, and fatty acid metabolism: $A C O X 1, A L D H 2$, and $H A D H B$ were up-regulated, and $M E 1$, $A C A C A$, and $F A S N$ were down-regulated under cold stress. $A C O X 1, A C A C A$ and $F A S N$ were discussed under the GO enrichment analysis. $H A D H B$ is a subunit of the mitochondrial trifunctional protein and has thiolase activity, which catalyzing the last three steps of mitochondrial beta-oxi- 
dation of long-chain fatty acids. ALDH2 encodes acetaldehyde dehydrogenase 2 , which catalyzes the oxidation of acetaldehyde to acetic acid. ME1 encodes malic enzyme 1, which catalyzes the oxidative decarboxylation of L-malate and NADP + to produce pyruvate and NADPH. NADPH is an important factor that influences the fatty acid synthesis. Based on our results, we speculate that the down-regulation of $M E 1, A C A C A$, and $F A S N$ reduces the activity of these enzymes, resulting in the production of acetyl CoA through fatty acid metabolism and propanoate metabolism. As a result, fatty acid synthesis is repressed. The up-regulated expression of $A C O X 1$ and $H A D H B$ indicates that fatty acid $\beta$-oxidation was enhanced under cold stress. Up-regulation of $A L D H 2$ increases the formation of acetic acid, which is further converted into acetyl coenzyme. This allows more acetyl coenzyme A to enters the Krebs cycle, releasing energy. This is consistent with the body's demand for energy during cold adaptation.

Taken together, our data reveal the mechanism of response to cold stress in broilers. These results may serve as an important reference for the selection and breeding of broilers and may provide new material for the chicken genomic studies.

\section{Acknowledgments}

This work was supported by the Natural Science Foundation of Heilongjiang Province (Grant No.C200932) and Heilongjiang Postdoctoral Financial Assistance (Grant No. LBH-Z10268).

\section{References}

Audic S and Claverie JM. The significance of digital gene expression profiles. Genome Research, 7: 986-995. 1997.

Baker L and Winegrad A. Fasting hypoglycaemia and metabolic acidosis associated with deficiency of hepatic fructose-1, 6diphosphatase activity. Lancet, 2: 13-16. 1970.

Benjamini Y and Hochberg Y. Controlling the false discovery rate: a practical and powerful approach to multiple testing. Journal of the Royal Statistical Society, Series B, 57: 289-300. 1995.

Chen X, Jiang R and Geng Z. Cold stress in broiler: global gene expression analyses suggest a major role of CYP genes in cold responses. Molecular Biology Reports, 39: 425-429. 2012.

Chen XY, Li R, Wang M and Geng ZY. Identification of differentially expressed genes in hypothalamus of chicken during cold stress. Molecular Biology Reports, 41: 2243-2248. 2014.

Cho KW, Morris DL, Delproposto JL, Geletka L, Zamarron B, Martinez -Santibanez G, Meyer KA, Singer K, O'Rourke RW and Lumeng CN. An MHC II dependent activation loop between adipose tissue macrophages and CD4 $+\mathrm{T}$ cells controls obesity-induced inflammation. Cell Reports, 9: 605-617. 2014.

Hangalapura BN, Kaiser MG, Poel JJ, Parmentier HK and Lamont SJ. Cold stress equally enhances in vivo pro-inflammatory cytokine gene expression in chicken lines divergently selected for antibody responses. Developmental and Comparative Immunology, 30: 503-511. 2006.

Hommes FA, Bendien K, Elema JD, Bremer HJ and Lombeck I. Two cases of phosphoenolpyruvate carboxykinase deficiency. Acta Paediatrica, 65: 233-240. 1976.

Jensen PE. Recent advances in antigen processing and presentation.
Nature Immunology, 8: 1041-1048. 2007.

Jump DB, Botolin D, Wang Y, Xu J, Christian B and Demeure O. Fatty acid regulation of hepatic gene transcription. Journal of Nutrition, 135: 2503-2506. 2005.

Jump DB, Clarke SD, Thelen A and Liimatta M. Coordinate regulation of glycolytic and lipogenic gene expression by polyunsaturated fatty acids. Journal of Lipid Research, 35: 10761084. 1994.

Kaufman J. The simple chicken major histocompatibility complex: life and death in the face of pathogens and vaccines. Philosophical Transactions of the Royal Society of London, Series B, Biological Sciences, 355: 1077-1084. 2000.

Langouet-Astrie CJ, Meinsen AL, Grunwald ER, Turner SD and Enke RA. RNA sequencing analysis of the developing chicken retina. Scientific Data, 3: 160117. 2016.

Leandro NS, Gonzales E, Ferro JA, Ferro MI, Givisiez PE and Macari M. Expression of heat shock protein in broiler embryo tissues after acute cold or heat stress. Molecular Reproduction and Development, 67: 172-177. 2004.

Leng L, Metz CN, Fang Y, Xu J, Donnelly S, Baugh J, Delohery T, Chen Y, Mitchell RA and Bucala R. MIF signal transduction initiated by binding to CD74. Journal of Experimental Medicine, 197: 1467-1476. 2003.

Li TT, Wang SY, Wu RM, Zhou XY, Zhu DH, Zhang Y. Identification of long non-protein coding RNAs in chicken skeletal muscle using next generation sequencing. Genomics, 5: 292298. 2012.

Livak KJ and Schmittgen TD. Analysis of relative gene expression data using real-time quantitative PCR and the $2^{-\Delta \Delta C T}$ method. Methods, 25: 402. 2001.

Miyazaki M, Sampath H, Liu X, Flowers MT, Chu K, Dobrzyn A and Ntambi JM. Stearoyl-CoA desaturase-1 deficiency attenuates obesity and insulin resistance in leptin-resistant obese mice. Biochemical and Biophysical Research Communications, 380: 818-822. 2009.

Morrissy AS, Morin RD, Delaney A, Zeng T, McDonald H, Jones S, Zhao Y, Hirst $M$ and Marra MA. Next-generation tag sequencing for cancer gene expression profiling. Genome Research, 19: 1825-1835. 2009.

Mujahid A and Furuse M. Oxidative damage in different tissues of neonatal chicks exposed to low environmental temperature. Molecular and Integrative Physiology, 152: 604-608. 2009.

Mutryn MF, Brannick EM, Fu W, Lee WR and Abasht B. Characterization of a novel chicken muscle disorder through differential gene expression and pathway analysis using RNAsequencing. BMC Genomics, 16: 399. 2015.

Nguyen P, Leray V, Diez M, Serisier S, Le Bloc'h J, Siliart B and Dumon H. Liver lipid metabolism. Journal of Animal Physiology and Animal Nutrition, 92: 272-283. 2008.

Nie QH, Sandford EE, Zhang XQ, Nolan LK, Lamont SJ. Deep Sequencing-Based Transcriptome Analysis of Chicken Spleen in Response to Avian Pathogenic Escherichia coli (APEC) Infection. PLoS One, 7: 41645. 2012.

Olanrewaju HA, Purswell JL, Collier SD and Branton SL. Effect of ambient temperature and light intensity on physiological reactions of heavy broiler chickens. Poultry Science, 89: 26682677. 2010.

Pan JQ, Tan X, Li JC, Sun WD and Wang XL. Effects of early feed restriction and cold temperature on lipid peroxidation, pulmonary vascular remodelling and ascites morbidity in broilers under normal and cold temperature. British Poultry Science, 46: 374-381. 2005. 
Ren $\mathrm{T}$ and Xin CA. The influence of cold stress on the chicken. Poultry Husbandry and Disease Control, 2: 32-33. 1997.

Rosen ED and Spiegelman BM. Adipocytes as regulators of energy balance and glucose homeostasis. Nature, 444: 847-853. 2006.

Sahin E and Gumuslu S. Cold-stress-induced modulation of antioxidant defence: role of stressed conditions in tissue injury followed by protein oxidation and lipid peroxidation. International Journal of Biometeorology, 48: 165-171. 2004.

Sampath $\mathrm{H}$ and Ntambi JM. Polyunsaturated fatty acid regulation of genes of lipid metabolism. Annual Review of Nutrition, 25: 317-340. 2005.

Smith M. Effects of feed intake and environmental temperature on chick growth and development. Journal of Agricultural Science, 121: 421-425. 1993.

Spinua M and Degena AA. Effect of cold stress on performance and immune responses of Bedouin and white leghorn hens. British
Poultry Science, 34: 177-185. 1993.

Stumptner-Cuvelette $\mathrm{P}$ and Benaroch P. Multiple roles of the invariant chain in MHC Class II function. Biochimica et Biophysica Acta, 1542: 1-13. 2002

Tobin DM, Jr Vary JC, Ray JP, Walsh GS, Dunstan SJ, Bang ND, Hagge DA, Khadge S, King MC, Hawn TR, Moens CB, and Ramakrishnan L. The 1ta4h locus modulates susceptibility to mycobacterial infection in zebrafish and humans. Cell, 140: 717-730. 2010.

Wang JT, Li S, Li JL, Zhang JW and Xu SW. Effects of cold stress on the messenger ribonucleic acid levels of peroxisome proliferator-activated receptor- $\gamma$ in spleen, thymus, and bursa of fabricius of chickens. Poultry Science, 88: 2549-2554. 2009.

Yao J, Wang Y, Wang W, Wang N, Li H. Solexa sequencing analysis of chicken pre-adipocyte microRNAs. Biosicience, Biotechnology, and Biochemistry, 75: 54-61. 2011. 